\title{
Celecoxib in lymphangioleiomyomatosis: results of a phase I clinical trial
}

\author{
To the Editor:
}

Lymphangioleiomyomatosis (LAM) is a multisystem disease associated with progressive pulmonary disease that affects almost exclusively women. LAM is characterised pathologically by proliferation of abnormal smooth muscle-like cells carrying mutations in predominantly the tuberous sclerosis complex (TSC) gene TSC2 and, rarely, in TSC1 [1]. LAM can occur sporadically or in association with TSC. Mutations in the TSC genes lead to activation of the mammalian/mechanistic target of rapamycin complex 1 (mTORC1) [2]. In the landmark randomised controlled MILES (Multicenter International LAM Efficacy of Sirolimus) trial, the mTORC1 inhibitor rapamycin stabilised lung function and improved symptoms in LAM patients with moderate to severe changes in lung function (forced expiratory volume in $\left.1 \mathrm{~s}\left(\mathrm{FEV}_{1}\right)<70 \%\right)$ [3]. Recent in vitro and preclinical evidence showed that loss of TSC2 resulted in upregulation of COX-2 and prostacyclin synthase (PGTIS) expression, independent of mTORC1. Treatment of $T s c 2^{+/-}$mice with celecoxib, a COX-2 specific inhibitor, resulted in a 50\% decrease in renal cystadenomas volume, which occur spontaneously in this model [4]. In addition, LAM nodules were found to express higher levels of COX-2 in comparison with control lungs [4].

Based on these data we performed the COX-2 inhibition in LAM clinical trial (COLA; NCT02484664), a phase I safety study of a single daily dose of celecoxib $(200 \mathrm{mg}$ ) taken orally in treatment-naïve patients with LAM who were deemed not to require treatment with mTORC1 inhibitors. The primary endpoint of the study was safety and tolerability. Toxicities were graded using the National Cancer Institute (NCI) Common Toxicity Criteria (version 4). The study design consisted of a screening visit and 24-week treatment period, with scheduled visits at weeks 8, 16 and end of study. Secondary endpoints included effects on lung function, quality of life, renal angiomyolipoma size, serum vascular endothelial growth factor (VEGF)-D levels, and levels of TSC2 mutation in plasma cell free-DNA (cfDNA).

From June 2016 through November 2018, 41 subjects were screened, 26 did not meet inclusion/exclusion criteria and 12 subjects provided written, informed consent to participate in the study and were enrolled on the study drug. Mean age of study participants was 48 (range 39-63) years. Six subjects were post-menopausal. No dose-limiting toxicities were encountered. A total of 75 non-serious adverse events were reported for 11 patients. No serious adverse events were reported; most non-serious adverse events (97\%, 73 out of 75$)$ were rated as mild (CTCAE grade 1). Nearly half (37/75) were thought to be related to celecoxib. The most commonly reported non-serious adverse events were stomach/abdominal pain (six events from three subjects) and headaches (six events from three subjects)

Three patients had their participation in the study ended early. One subject withdrew due to gastrointestinal side-effects probably related to celecoxib; one subject was withdrawn due to a rash probably related to celecoxib; and one subject withdrew due to a cardiovascular event, likely unrelated to celecoxib.

A secondary objective of the COLA trial was to determine the effects of celecoxib on lung function. The least square mean of post-bronchodilator forced vital capacity (FVC) (mL), post-bronchodilator $\mathrm{FEV}_{1}$ and diffusing capacity of the lung for carbon monoxide $\left(D_{\mathrm{LCO}}\right)$ did not change significantly during the study period. The results were similar when a subgroup analysis of pre-menopausal subjects $(n=6)$ was conducted. This is not surprising, considering the short course of the study, 24 weeks, and mild lung disease in the enrolled subjects. Whether this reflects an effect of celecoxib in reducing lung function

@ERSpublications

COX2 inhibition is safe in LAM patients with mild disease. In the subset of patients with high VEGF-D ( $>800 \mathrm{pg}$ per $\mathrm{mL}$ ) COX2 inhibition appears to cause a decrease in VEGF-D levels and may provide clinical benefit. http://bit.ly/395drXs

Cite this article as: El-Chemaly S, Taveira-DaSilva A, Bagwe S, et al. Celecoxib in lymphangioleiomyomatosis: results of a phase I clinical trial. Eur Respir J 2020; 55: 1902370 [https://doi. org/10.1183/13993003.02370-2019]. 
decline requires studies with larger enrolled populations and longer-term treatment. Over the study period, we found no change in the St George's Respiratory Questionnaire, a validated tool to assess quality of life in patients with LAM $[5,6]$ (table 1$)$.

Baseline magnetic resonance imaging (MRI) showed a kidney angiomyolipoma in three subjects, which remained unchanged at the end of the study. Additional findings on screening MRI included kidney cysts $(\mathrm{n}=5$ subjects), renal haemorrhagic or preoteinaceous cysts $(\mathrm{n}=2)$, liver haemangiomas $(\mathrm{n}=3)$, liver cysts $(n=5)$, vertebral haemangioma $(n=2)$, and abdominal lymphangiomyomas $(n=1)$. Seven subjects had a repeat MRI, and no significant changes were observed except in one subject with bilateral renal cysts, which decreased in size. Four other subjects had renal cysts that did not change in size during the treatment period.

At screening visit, median serum VEGF-D levels were 448 (range 279-2232) pg.mL ${ }^{-1}$. Two out of 12 subjects had VEGF-D levels greater than $800 \mathrm{pg} \cdot \mathrm{mL}^{-1}$. Treatment with celecoxib for 24 weeks did not result in significant changes in mean VEGF-D (table 1). However, the two subjects who had VEGF-D levels higher than $800 \mathrm{pg} \cdot \mathrm{mL}^{-1}$ (meeting diagnostic criteria for LAM) demonstrated significant reductions in VEGF-D levels at the end of treatment with a decrease from baseline of 2156 and $2232 \mathrm{pg} \cdot \mathrm{mL}^{-1}$ to 1408 and $1865 \mathrm{pg} \cdot \mathrm{mL}^{-1}$, respectively. Further, $D_{\mathrm{LCO}}$ and post-bronchodilator $\mathrm{FEV}_{1}$ and FVC remained stable in these two subjects. Serum VEGF-D levels have been shown previously to correlate with disease severity and response to mTOR inhibition [7]. Previous studies have shown that VEGF-D can regulate prostaglandin biosynthesis and COX mRNA expression [8]. Further, COX-2 inhibition has been shown to inhibit nucleolin, which results in decreased VEGF-D mRNA translation [9]. Hence, these very preliminary findings ( $\mathrm{n}=2$ subjects) of a drop in VEGF-D suggest the possibility that COX-2 inhibition may have therapeutic benefit in patients with early stage LAM and elevated VEGF-D levels. There was no difference in PGEM (a stable metabolite of prostaglandin $\mathrm{E}_{2}$ ) plasma levels between screening (7.5 (range 5.4-8.7) $\left.\mathrm{pg} \cdot \mathrm{mL}^{-1}\right)$ and end of study or early termination visits (7 (range 4.4-13.4) $\mathrm{pg} \cdot \mathrm{mL}^{-1}$ ) (paired t-test 0.7). This result could be due to potential under-dosing [10], or pharmacokinetics of celecoxib, which has a half-life of 8-12 h [11], since plasma was collected $24 \mathrm{~h}$ after last celecoxib dose.

Plasma cfDNA samples were collected from all COLA subjects and subjected to high read depth massively parallel sequencing analysis of TSC1/TSC2 (mean read depth: 1040×) [12]. Although several candidate mutation findings were identified, they did not replicate in a validation analysis. Hence, there were no mutation findings in either TSC1 or TSC2 in plasma cfDNA from these subjects. These data are consistent with our recent analysis of a larger LAM cohort $(n=61)[12]$.

The use of COX-2 inhibitors as preventive therapy for cancer in healthy individuals is counterbalanced by potential adverse cardiovascular events [13]. However, as an adjunct therapy in cancer treatment or prevention of recurrence, COX-2 inhibition remains a subject of intense investigation. The clinicaltrials.gov trial registry shows more than a thousand studies examining the COX-2 pathway in a variety of cancers, with more than 200 using celecoxib, some with significantly higher doses than used in this study. This is the first study to examine COX-2 inhibition in lung disease of any kind, to our knowledge.

Our study raises important questions: 1) are women with LAM with low burden of disease and high VEGF-D levels candidates for celecoxib treatment? Should this subgroup of patients be the target of subsequent clinical trials? 2) Could COX-2 inhibition play a role in the prevention of LAM development in patients with TSC? 3) Is there a role for celecoxib in addition to mTORC1 inhibition in the treatment of LAM and potentially other manifestations of TSC?

\begin{tabular}{|c|c|c|c|}
\hline Variable & Baseline & End of treatment ${ }^{\#}$ & $\mathrm{p}$-value \\
\hline $\mathrm{FEV}_{1}^{\pi} \mathrm{mL}$ & $2583 \pm 166$ & $2518 \pm 166$ & 0.14 \\
\hline $\mathrm{FVC}^{\pi} \mathrm{mL}$ & $3415 \pm 197$ & $3384 \pm 197$ & 0.45 \\
\hline$D_{\mathrm{Lco}} \mathrm{mL} \cdot \mathrm{mmHg}^{-1} \cdot \mathrm{min}^{-1}$ & $18.6 \pm 1.1$ & $18.4 \pm 1.1$ & 0.24 \\
\hline SGRQ & $19.1 \pm 4.9$ & $21.3 \pm 4.9$ & 0.20 \\
\hline VEGF-D & $755 \pm 199$ & $656 \pm 143$ & 0.17 \\
\hline
\end{tabular}

Data are presented as least square mean \pm SEM, unless otherwise stated. $\mathrm{FEV}_{1}$ : forced expiratory volume in $1 \mathrm{~s}$; FVC: forced vital capacity; $D_{\mathrm{LCO}}$ : diffusing capacity of the lung for carbon monoxide; SGRQ: St George's Respiratory Questionnaire; VEGF: vascular endothelial growth factor. \#: end of study visit or early termination; ${ }^{\text {ๆ: }}$ post-bronchodilators. 
In summary, the COLA trial has established the safety of celecoxib $200 \mathrm{mg}$ orally daily in patients with LAM. Larger phase II/III trials are needed to establish the effectiveness of this treatment, perhaps in a subset of patients with LAM and high VEGF-D levels.

Souheil El-Chemaly ${ }^{1,5}$, Angelo Taveira-DaSilva ${ }^{2}$, Shefali Bagwe ${ }^{1}$, Katarzyna Klonowska ${ }^{1}$, Tania Machado ${ }^{2}$, Anthony M. Lamattina ${ }^{1}$, Hilary J. Goldberg ${ }^{1}$, Amanda M. Jones ${ }^{2}$, Patricia Julien-Williams ${ }^{2}$, Rie Maurer ${ }^{3}$, Ivan O. Rosas ${ }^{1,4}$, Elizabeth P. Henske ${ }^{1}$, Joel Moss ${ }^{2}$ and David J. Kwiatkowski ${ }^{1,5}$

${ }^{1}$ Division of Pulmonary and Critical Care Medicine, Brigham and Women's Hospital, Harvard Medical School, Boston, MA, USA. ${ }^{2}$ Pulmonary Branch, National Heart, Lung, and Blood Institute, National Institutes of Health, Bethesda, MD, USA. ${ }^{3}$ The Harvard Clinical and Translational Science Center, Boston, MA, USA. ${ }^{4}$ Pulmonary, Critical Care and Sleep Medicine, Baylor College of Medicine, Houston, TX, USA. ${ }^{5}$ Equal contribution.

Correspondence: David J. Kwiatkowski, Cancer Genetics Laboratory, Division of Pulmonary and Critical Care Medicine, Brigham and Women's Hospital, 75 Francis Street, Boston, MA 02115, USA. E-mail: dk@rics.bwh.harvard.edu

Received: 3 Dec 2019 | Accepted after revision: 2 Feb 2020

Acknowledgement: We are indebted to patients and their families for their participation in this study. We also thank The LAM Foundation and the Tuberous Sclerosis Alliance for patient referral. We thank the Data Safety Monitoring Board Members and Medical Monitor, as well as the clinical trials support staffs at the BWH and the Office of the Clinical Director, National Heart, Lung, and Blood Institute, NIH. We are grateful to Saint George's, University London for permission to use the SGRQ.

This study is registered as clinical trial NCT02484664. Data generated in this study will be made available in de-identified fashion upon reasonable request and with appropriate institutional review board approvals.

Conflict of interest: S. El-Chemaly has nothing to disclose. A. Taveira-DaSilva has nothing to disclose. S. Bagwe has nothing to disclose. K. Katarzyna has nothing to disclose. T. Machado has nothing to disclose. A.M. Lamattina reports personal fees from Wave Life Sciences, outside the submitted work. H.J. Goldberg has nothing to disclose. A.M. Jones has nothing to disclose. P. Julien-Williams has nothing to disclose. R. Maurer has nothing to disclose. I.O. Rosas has nothing to disclose. E.P. Henske has nothing to disclose. J. Moss has nothing to disclose. D.J. Kwiatkowski has nothing to disclose.

Support statement: This study was supported by a Department of Defense Grant W81XWH-15-1-0511, TS140054 to D.J. Kwiatkowski and in part by the Division of Intramural Research NIH/NHLBI and the Lucy Engles Program in TSC and LAM research. Funding information for this article has been deposited with the Crossref Funder Registry.

\section{References}

1 McCormack FX, Henske EP. Lymphangioleiomyomatosis and pulmonary disease in tsc. In: Kwiatkowski DJ, Whittemore VH, Thiele EA, eds. Tuberous Sclerosis Complex. Weinheim, Wiley-VCH, 2010; pp. 345-368.

2 Kwiatkowski DJ, Manning BD. Tuberous sclerosis: a gap at the crossroads of multiple signaling pathways. Hum Mol Genet 2005; 14: R251-R258.

3 McCormack FX, Inoue Y, Moss J, et al. Efficacy and safety of sirolimus in lymphangioleiomyomatosis. N Engl J Med 2011; 364: 1595-1606.

4 Li C, Lee PS, Sun Y, et al. Estradiol and MTORC2 cooperate to enhance prostaglandin biosynthesis and tumorigenesis in TSC2-deficient LAM cells. J Exp Med 2014; 211: 15-28.

5 Swigris JJ, Lee HS, Cohen M, et al. St. George's respiratory questionnaire has longitudinal construct validity in lymphangioleiomyomatosis. Chest 2013; 143: 1671-1678.

6 El-Chemaly S, Taveira-Dasilva A, Goldberg HJ, et al. Sirolimus and autophagy inhibition in lymphangioleiomyomatosis: results of a phase I clinical trial. Chest 2017; 151: 1302-1310.

7 Young L, Lee HS, Inoue Y, et al. Serum VEGF-D a concentration as a biomarker of lymphangioleiomyomatosis severity and treatment response: a prospective analysis of the Multicenter International Lymphangioleiomyomatosis Efficacy of Sirolimus (MILES) trial. Lancet Respir Med 2013; 1: 445-452.

8 Davydova N, Harris NC, Roufail S, et al. Differential receptor binding and regulatory mechanisms for the lymphangiogenic growth factors vascular endothelial growth factor (VEGF)-C and -D. J Biol Chem 2016; 291: 27265-27278.

9 Morfoisse F, Tatin F, Hantelys F, et al. Nucleolin promotes heat shock-associated translation of VEGF-D to promote tumor lymphangiogenesis. Cancer Res 2016; 76: 4394-4405.

10 Yang YC, Lu ML, Rao JY, et al. Joint association of polymorphism of the fgfr4 gene and mutation tp53 gene with bladder cancer prognosis. Br J Cancer 2006; 95: 1455-1458.

11 Werner $\mathrm{U}$, Werner $\mathrm{D}$, Pahl A, et al. Investigation of the pharmacokinetics of celecoxib by liquid chromatography-mass spectrometry. Biomed Chromatogr 2002; 16: 56-60.

12 Ogorek B, Hamieh L, Lasseter $\mathrm{K}$, et al. Generalised mosaicism for tsc2 mutation in isolated lymphangioleiomyomatosis. Eur Respir J 2019; 54: 1900938.

13 McCormack PL. Celecoxib: a review of its use for symptomatic relief in the treatment of osteoarthritis, rheumatoid arthritis and ankylosing spondylitis. Drugs 2011; 71: 2457-2489. 